02,13

\title{
Спектральный отклик матриц полуволновых и электрически малых антенн с СИНИС-болометрами
}

\author{
(C) А.А. Гунбина, М.А. Тарасов, С.А. Лемзяков, А.М. Чекушкин, Р.А. Юсупов, Д.В. Нагирная, \\ М.А. Мансфельд, В.Ф. Вдовин, Д. Винклер, А.С. Калабухов, С. Махашабде, В.С. Эдельман \\ Институт радиотехники и электроники им. В.А. Котельникова РАН, \\ Москва, Россия \\ E-mail: aleksandragunbina@mail.ru
}

Поступила в Редакцию 26 марта 2020 г.

В окончательной редакции 26 марта 2020 г.

Принята к публикации 2 апреля 2020 г.

\begin{abstract}
Разработаны, изготовлены и исследованы две серии матриц кольцевых полуволновых и электрически малых антенн с характерными размерами элементов, соответствующими 1/10 длины волны субтерагерцевого диапазона с интегрированными болометрами структуры сверхпроводник-изолятор-нормальный металлизолятор-сверхпроводник (СИНИС). Для повышения точности оценки спектрального отклика приемных матриц необходимо проводить численное моделирование всей структуры, а в экспериментальных исследованиях вводить дополнительные опорные каналы. В настоящей работе для нормировки использовались три опорных канала: пироэлектрический приемник снаружи криостата и два холодных канала - болометр $\mathrm{RuO}_{2}$ и цепочка СИН-переходов в качестве термометра.
\end{abstract}

Ключевые слова: СИНИС-болометры, матрицы планарных антенн, терагерцевое излучение.

DOI: $10.21883 /$ FTT.2020.09.49767.35H

\section{1. Введение}

В связи с возросшим в последние годы интересом к астрономическим наблюдениям в миллиметровом и субмиллиметровом диапазоне [1] появилась необходимость создания высокочувствительных приемных систем на основе охлаждаемых болометров. К таким системам предъявляются жесткие и противоречивые требования - мощность эквивалентная шуму (МЭШ) не хуже $10^{-16} \mathrm{~W} / \mathrm{Hz}^{1 / 2}$ для наземных обсерваторий и на три порядка лучше для космических при широком динамическом диапазоне для работы в условиях сравнительно высокой фоновой мощности (десятки pW для наземных обсерваторий). Наиболее развитыми и широко используемыми на сегодня являются болометры на краю сверхпроводящего перехода (БКП) [2,3] и болометры на кинетической индуктивности (КИД) [4,5]. БКП имеют высокую чувствительность, но узкий динамический диапазон, низкое быстродействие и необходимость поддержания рабочей температуры с высокой точностью. Главным достоинством КИДов является низкий уровень собственных шумов в силу отсутствия шума Найквиста, но абсолютная величина отклика кинетической индуктивности мала. Эти недостатки отсутствуют в предлагаемых болометрах структуры сверхпроводник-изолятор-нормальный метал-изолятор-сверхпроводник (СИНИС) [6,7]. Одиночный СИНИС-болометр насыщается на уровне мощности менее $1 \mathrm{pW}$. В связи с этим, такие болометры объединяются в матрицы, в которых энергия сигнала, собираемого зеркалом телескопа, распределяется между болометрами. МЭШ разработанных структур - не хуже $10^{-16} \mathrm{~W} / \mathrm{Hz}^{1 / 2}$, но может быть улучшена путем модернизации системы считывания, как минимум, на порядок путем замены комнатных усилителей на холодные и применением микроволнового считывания.

Были разработаны и исследованы две серии приемных матриц кольцевых антенн в широком диапазоне частот $(60-600 \mathrm{GHz})$ : одна, состоящая из антенн диаметром $300 \mu \mathrm{m}$, рассчитана как обычная полуволновая антенна на центральную частоту $350 \mathrm{GHz}$; вторая матрица - из антенн диаметром 50-60 $\mu \mathrm{m}$ - электрически мала для этой частоты (матрица в конфигурации метаматериалов). Первые экспериментальные результаты с матрицами „электрически малых антенн“ были получены при облучении матрицы полуволновых антенн (рассчитанных на центральную частоту $350 \mathrm{GHz}$ ) диаметром $300 \mu \mathrm{m}$ на существенно более низкой частоте $60 \mathrm{GHz}$. Вольтваттная чувствительность оказалась достаточно высокой, при этом спектральная характеристика стала более равномерной. Это натолкнуло на решение пропорционально уменьшить диаметр колечек для приема сигнала на $350 \mathrm{GHz}$, т.е. до $50 \mu \mathrm{m}$, что и было реализовано в наших матрицах метаматериалов для трех типов антенн диаметром 50-60 $\mathrm{m}$ [7].

Для повышения точности оценки спектрального отклика таких матриц необходимо проводить моделирование всей структуры, а в экспериментальных исследованиях применять дополнительную калибровку принимаемого сигнала.

\section{2. СИНИС-болометр}

Чувствительным элементом такого болометра является абсорбер (поглотитель) из нормального металла. Два 
$a$

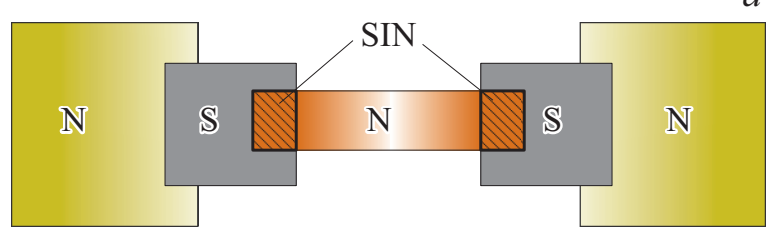

$b$
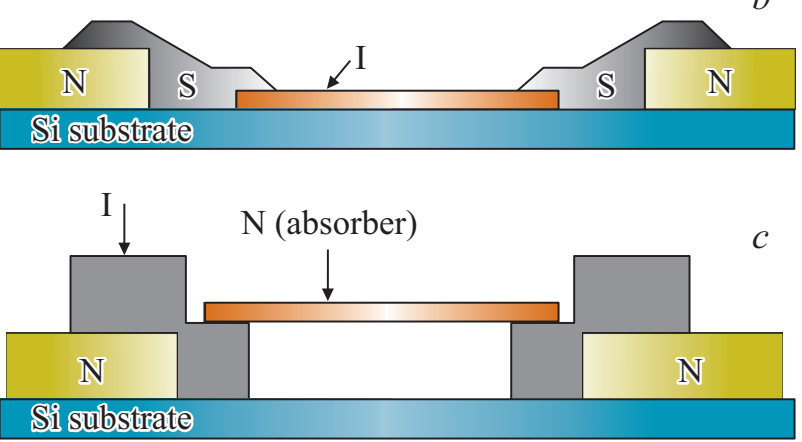

Рис. 1. Схематическое изображение СИНИС-болометра ( $\mathrm{N}$ - нормальный метал, I - изолятор, $\mathrm{S}$ - сверхпроводник): $a)$ вид сверху; $b$ ) вид сбоку СИНИС-болометра с поглотителем на подложке; $c$ ) вид сбоку СИНИС-болометра с подвешенным абсорбером.

СИН-перехода, соединенные последовательно с абсорбером, выступают в качестве термометров. Поглощение излучения в СИНИС-структурах вызывает разогрев абсорбера, который может быть зарегистрирован по увеличению туннельного тока СИН-термометров. Схематическое изображение СИНИС-болометра представлено на рис. 1. Оценка чувствительности в таких структурах опирается на неравновесные электронные и фононные функции распределения и механизмы квантового поглощения излучения [8]. Наиболее перспективным, для повышения чувствительности таких структур, является развитие СИНИС-болометров с подвешенным абсорбером [9], рис. 1, $c$.

\section{3. Матрицы полуволновых антенн}

В первых работах по исследованию матриц полуволновых кольцевых антенн диапазона $350 \mathrm{GHz}$ с интегрированными СИНИС-болометрами [10] был использован простейший подход: использование периодических граничных условий при моделировании матрицы, а в эксперименте - нормирование сигнала, снимаемого с болометрической матрицы, лишь на один опорный канал, расположенный снаружи криостата. Точность такой калибровки составляет около 50\%. Результаты моделирования и измерения в узкой полосе частот из работы [10] приведены на рис. 2. Параметр S11 (рис. 2,a) - это коэффициент передачи. При моделировании антенны, возбуждая порт в антенне, по рассчитанному параметpy S11 получаем амплитудно-частотную характеристику.
По теореме взаимности, антенна одинаково работает как на прием, так и на передачу, поэтому полученные данные могут быть использованы для оценки характеристик приемной антенны.

Максимально приблизить результаты моделирования к реальным условиям можно лишь путем моделирования целой матрицы с открытыми граничными условиями. Такой подход значительно увеличивает время счета, но дает более точный результат. Но и такой метод не даст полную электродинамическую картину т.к. в случае реальных условий существует огромное количество пространственных мод, а также множественные переотражения сигнала на пути от источника до приемной матрицы.

Эффективность поглощения приходящего сигнала существенно зависит от толщины подложки, на которой изготовлена матрица планарных антенн. Более подробно этот вопрос рассмотрен в работе [11]. На рис. 3 представлены результаты моделирования приемной матрицы полуволновых кольцевых антенн для трех различных толщин кремниевой подложки (диэлектрическая проницаемость 11.6): подложка толщиной $380 \mu \mathrm{m}$ (стандартная толщина подложки), $127 \mu \mathrm{m}-$ полуволновая подложка и $64 \mu \mathrm{m}-$ четвертьволновая подложка. Мода 1 и мода 2 - взаимно ортогональные поляризации. Различие объясняется влиянием соединительных проводов между элементами матрицы. Моделирование было сделано в программном пакете CST STUDIO SUITE с открытыми граничными условиями и круглым волноводом
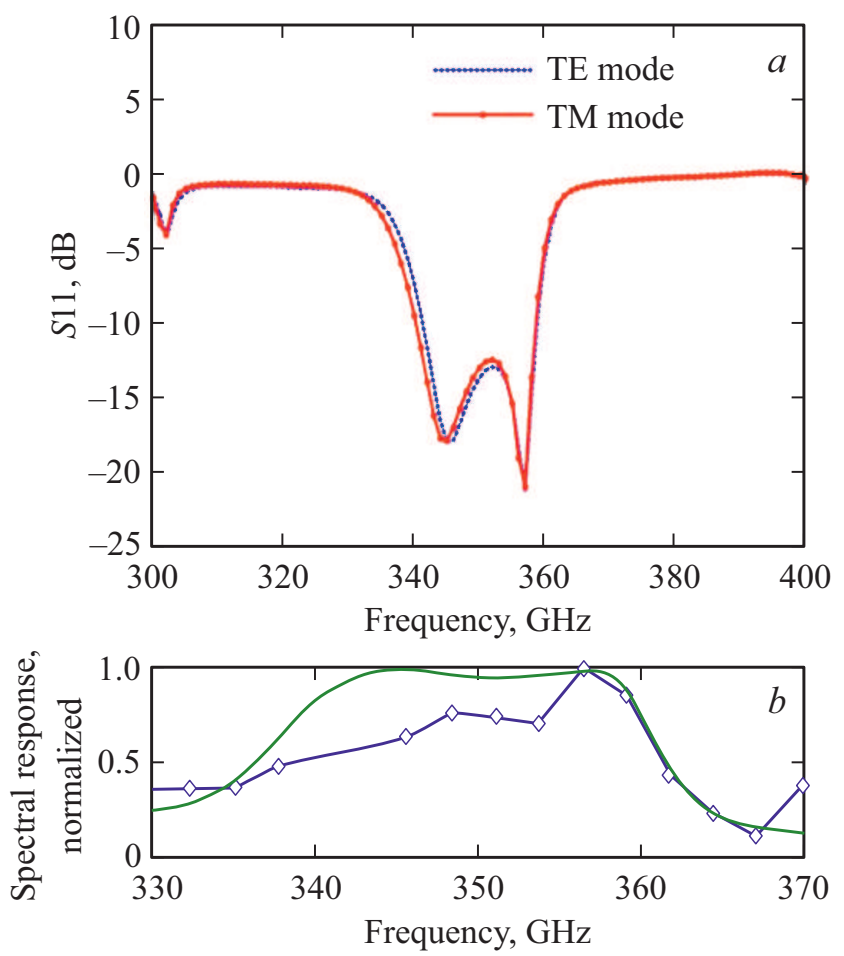

Рис. 2. Параметр $\mathrm{S} 11$ (a) и спектральный отклик $(b)$ в упрощенном случае из работы $[10]$; символы на части $b-$ экспериментально измеренный отклик. 

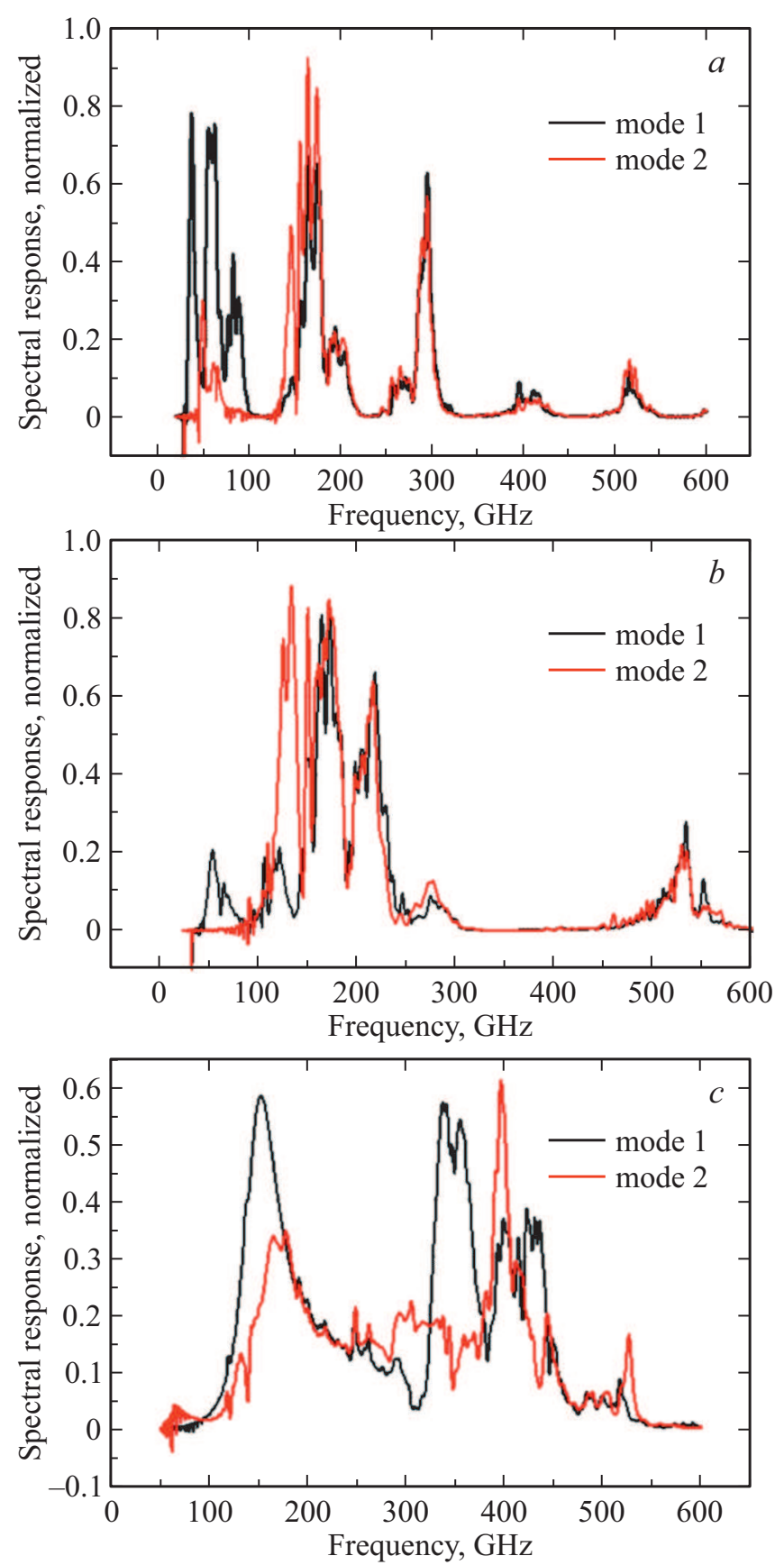

Рис. 3. Результаты моделирования матрицы полуволновых антенн диапазона $350 \mathrm{GHz}$ на подложках разной толщины: a) $380 \mu \mathrm{m}$, толщина стандартной кремниевой подложки; b) $127 \mu \mathrm{m}$, полуволновая подложка; c) $64 \mu \mathrm{m}$, четвертьволновая подложка.

в качестве направляющей структуры. Матрица состоит из 25-ти кольцевых антенн с двумя интегрированными СИНИС-болометрами. В проекте болометр представляет собой последовательное соединение дискретного порта (сопротивление абсорбера - 50 $\Omega$ ) и сосредоточенного элемента (емкость СИН-переходов болометpa $25 \mathrm{fF}$ ). Элементы матрицы могут быть соединены как последовательно, так и параллельно, в зависимости от требуемого выходного сопротивления для согласования с системой считывания. Все исследуемые структуры, представленные в данной работе, облучаются приходящим сигналом со стороны антенн, а на обратную сторону подложки наносится контррефлектор в виде толстой $(200 \mathrm{~nm})$ пленки Тi/Au. Различные методы облучения приемных матриц (со стороны диэлектрической подложки и со стороны антенн) более подробно рассмотрены в $[11,12]$.

\section{4. Матрицы электрически малых антенн (метаматериалы)}

Матрица элементов с размерами и периодом много меньше длины волны (электрически малые антенны) может эффективно взаимодействовать с электромагнитным излучением. Разрезанный кольцевой резонатор может служить классическим примером таких структур [13]. Для сравнения, на рис. 4, представлены фотографии единичных элементов матриц полуволновых и электрически малых кольцевых антенн диапазона $350 \mathrm{GHz}$, разрабатываемых нашим коллективом.

Расчет матриц электрически малых антенн был сделан в трех приближениях. Первые два (упрощенные модели): аналитическая модель сосредоточенных элементов и численная модель бесконечной матрицы с периодическими граничными условиями для каждой одиночной ячейки [7,14]. В нашем дизайне внешний диаметр кольца $R$ равен $54 \mu \mathrm{m}$ и ширина кольца $r$ равна $15 \mu \mathrm{m}$, что дает индуктивность для пленки из золота $L=\mu_{0} R[\ln (8 R / r)-1.75]=54.6 \mathrm{pH}$ (здесь $\mu_{0}-$ магнитная проницаемость вакуума).

Для алюминиевых СИН-переходов характерная емкость на $1 \mu \mathrm{m}^{2}$ составляет $70 \mathrm{fF}$. Для четырех СИНИС-болометров с восемью СИН-переходами площадью $0.8 \mu \mathrm{m}^{2}$, расположенных последовательно, получаем емкость $7 \mathrm{fF}$. C точки зрения постоянного тока, мы имеем две пары параллельных СИНИС-болометров. Но для приходящего СВЧ-излучения наше кольцо представляет собой независимую элементарную ячейку, в которой последовательно по кругу включены четыpe СИНИС-болометра. Коэффициент качества такого приемника при характерном сопротивлении болометра $30 \Omega$ составляет $Q=\omega L / R=3$. При этом резонансная частота равна $257 \mathrm{GHz}$. Подобный расчет был сделан для первоначальной оценки. Численное моделирование с использованием периодических граничных условий (как было сделано ранее в [7,14]) подходит лишь для расчета фазированных антенных решеток. Полученная гладкая зависимость (рис. 5,a) подтверждает, что такой подход может быть использован лишь для начальных приближений.

Для более точной оценки спектрального отклика использовали третий метод - численное моделирование целой структуры (100 кольцевых антенн с интегрированными болометрами) с открытыми граничными усло- 

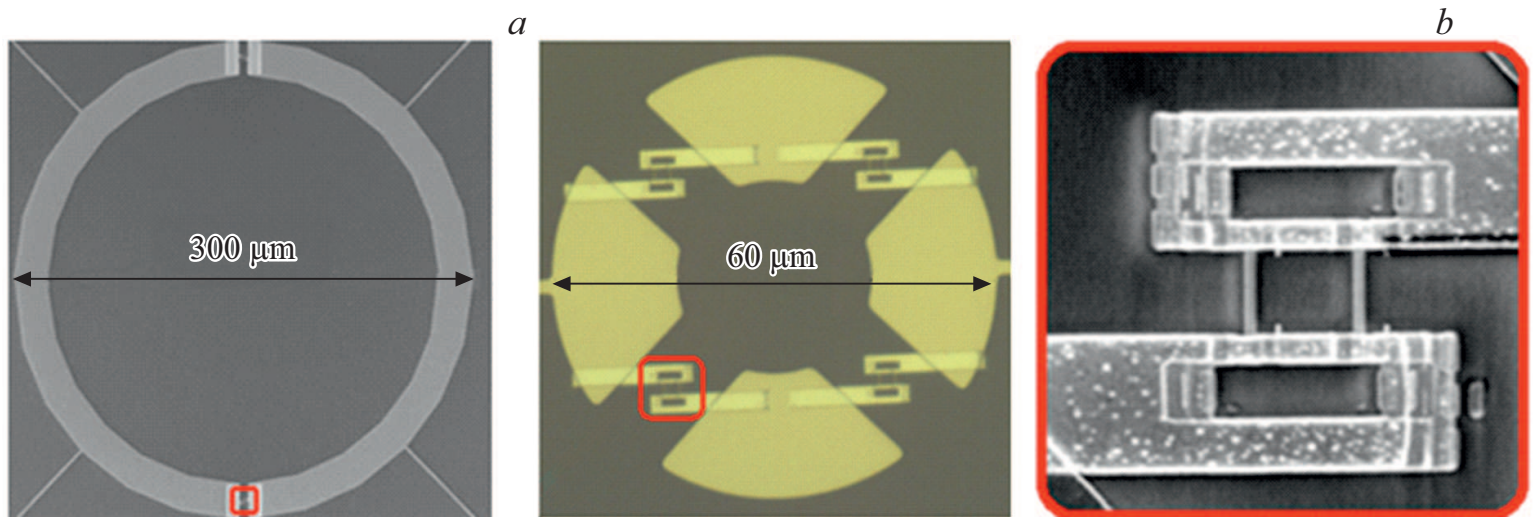

Рис. 4. Единичные элементы разрабатываемых матриц диапазона $350 \mathrm{GHz}:$ ) полуволновая антенна (слева) и электрически малая антенна (справа); b) СИНИС-болометр, интегрированный в антенны [12].

виями и источником излучения (волноводный порт) в дальней зоне. Результаты моделирования спектрального отклика такой структуры также представлены на рис. 5.
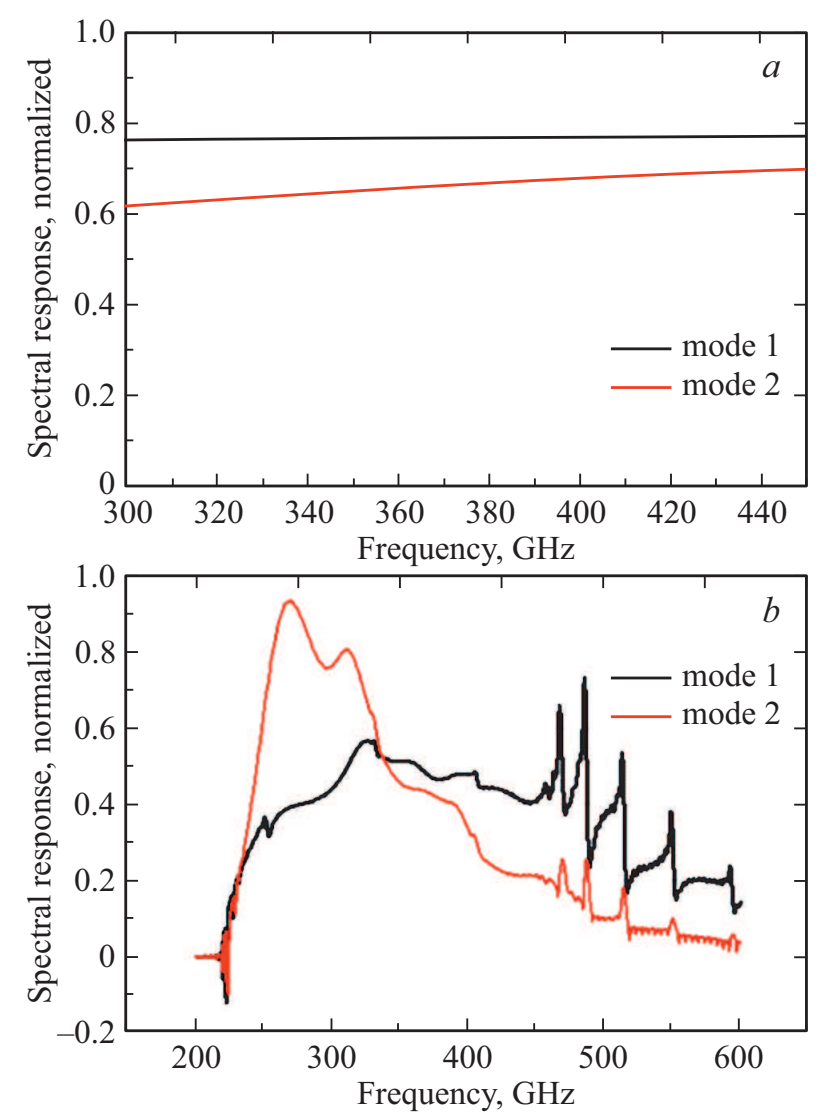

Рис. 5. Результаты моделирования матриц электрически малых антенн: $a$ ) упрощенная модель с периодическими граничными условиями, $b$ ) моделирование полной матрицы из 100 электрически малых антенн. Как и для матриц полуволновых антенн, различие между модами 1 и 2 объясняется наличием соединительных проводов между элементами. На части $b$ ) резкий спад ниже $220 \mathrm{GHz}$ определяется диаметром волновода, используемого в качестве направляющей структуры.

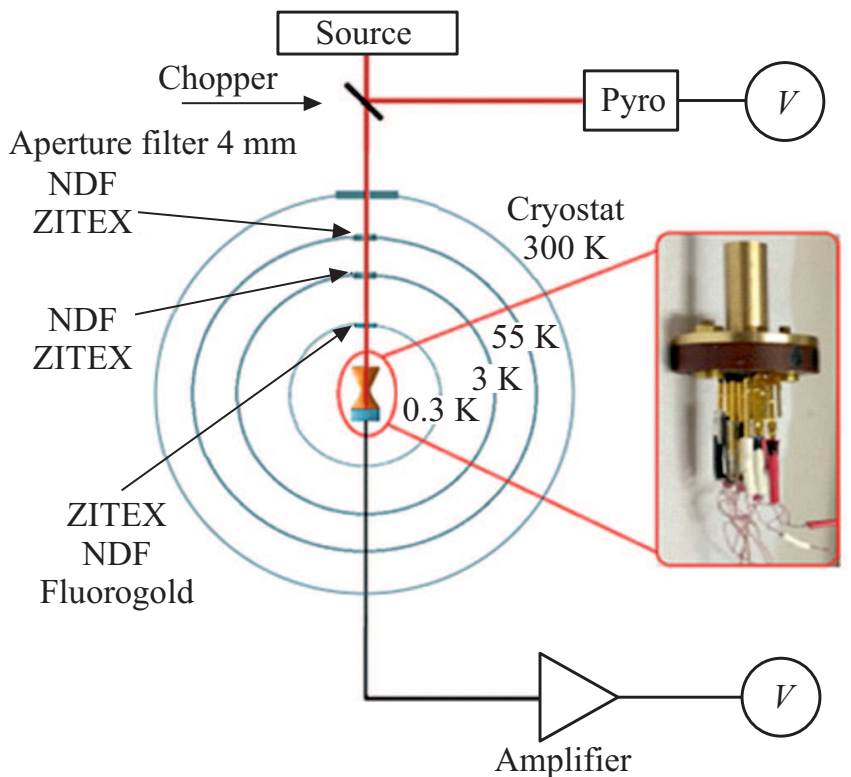

Рис. 6. Ранее используемая экспериментальная установка с одним опорным каналом снаружи криостата (пироэлектрический приемник) для измерения спектрального отклика приемной матрицы [12].

\section{5. Экспериментальная установка и результаты измерений}

Экспериментальное исследование спектрального отклика разрабатываемых структур при низких температуpax проводилось в криостате фирмы Oxford Instruments Heliox AC-V. Исследуемый образец устанавливался на холодную плиту $(280 \mathrm{mK})$ криостата. В качестве источника излучения использовали лампу обратной волны (ЛОВ) диапазона $240-370 \mathrm{GHz}$. В предыдущих работах использовалась калибровка сигнала источника только на один опорный канал, расположенный снаружи криостата (рис. 6). Точность такой калибровки составляет не более $50 \%$, учитывается лишь уровень 

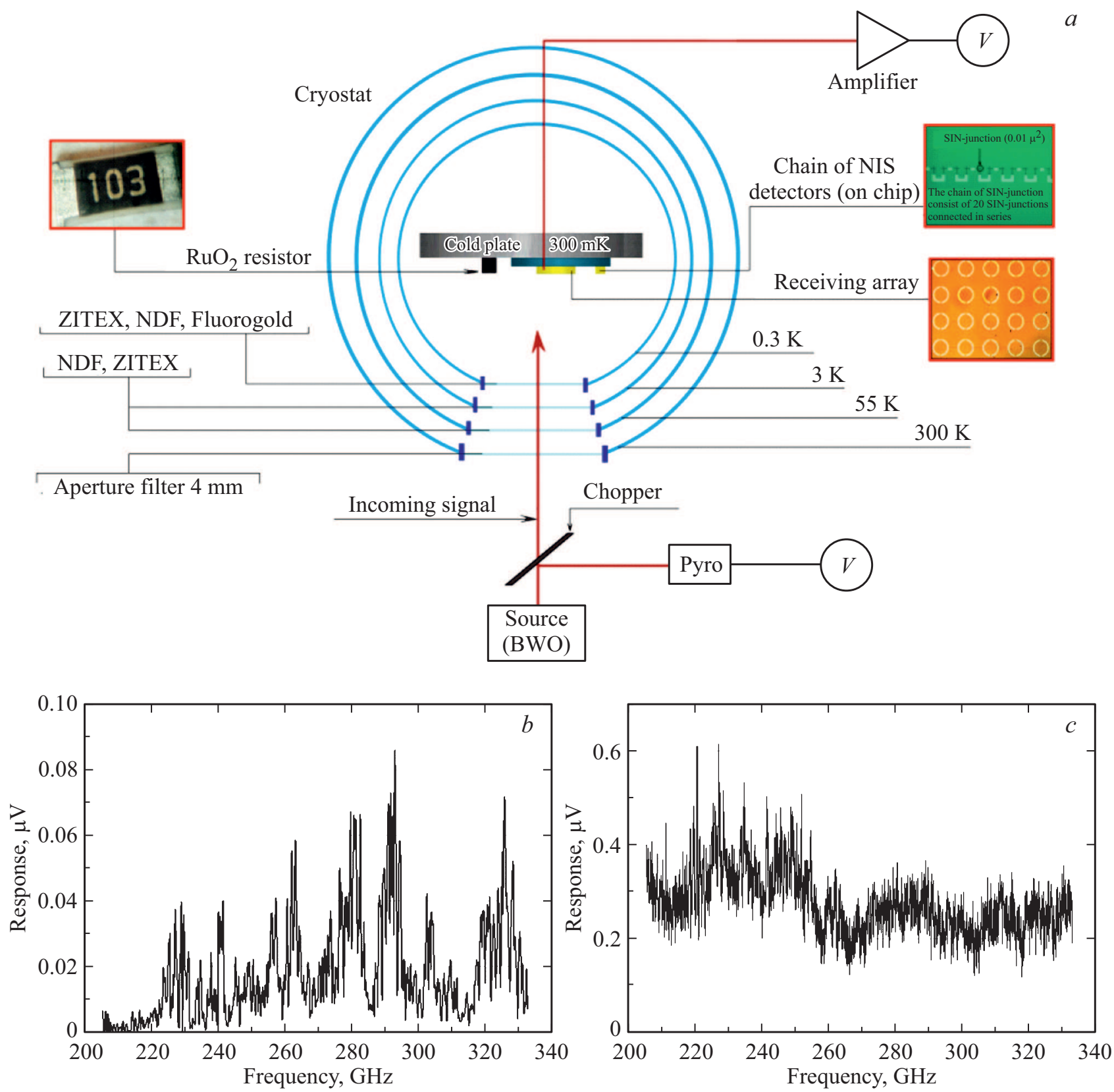

Рис. 7. Экспериментальная установка с тремя опорными каналами: $a$ ) схематическое изображение экспериментальной установки; b) сигнал, измеренный с рутениевого резистора; c) сигнал, измеренный с цепочки СИН-переходов.

сигнала источника снаружи криостата. Такой подход не позволяет учесть множественные переотражения на окнах криостата и внутри него. Для повышения точности оценки спектрального отклика мы ввели еще два дополнительных опорных канала внутри криостата: рутениевый резистор [15], расположенный на холодной плите криостата рядом с исследуемым образцом, и цепочку СИН-структур на самом исследуемом образце. Экспериментальная установка с тремя опорными каналами представлена на рис. 7, $a$. Измеренные сигналы с рутениевого резистора и цепочки СИН-переходов приведены на рис. 7, $b$ и 7, $c$ соответственно. Измеренный и нормированный на три опорных канала спектральный отклик матриц полуволновых и электрически малых антенн представлен на рис. 8 .

\section{6. Обсуждение результатов}

Как было сказано выше, оценка спектрального отклика приемной матрицы на основе охлаждаемых болометров - сложная комплексная задача. Помимо переотражений на пути „источник-приемник“ сигнал источника (ЛОВ) довольно нестабилен, и с изменением ускоряющего напряжения (и, соответственно, генерируемой частоты), мощность сигнала сильно изменяется. 

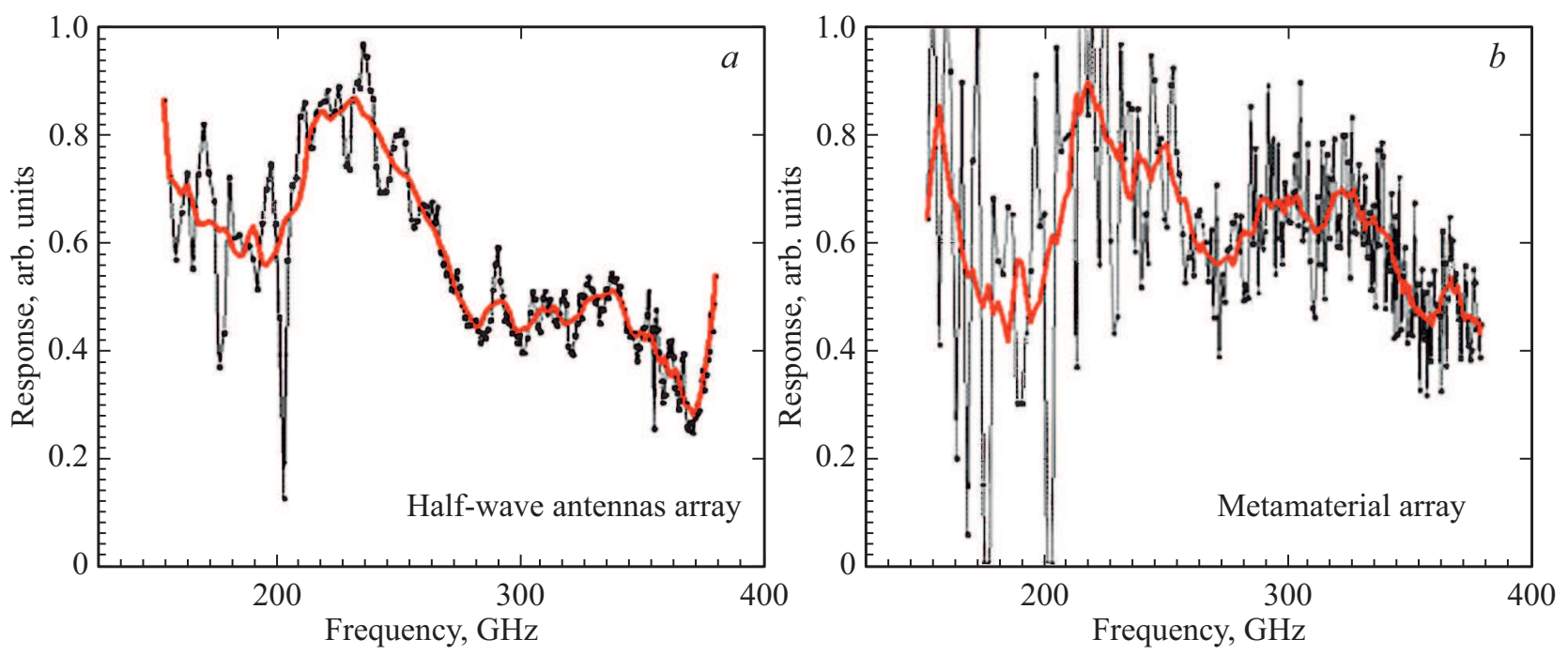

Рис. 8. Измеренный и нормированный на три опорных канала спектральный отклик матриц кольцевых планарных антенн диапазона $350 \mathrm{GHz}: a$ ) матрица полуволновых антенн, $b$ ) матрица электрически малых антенн.

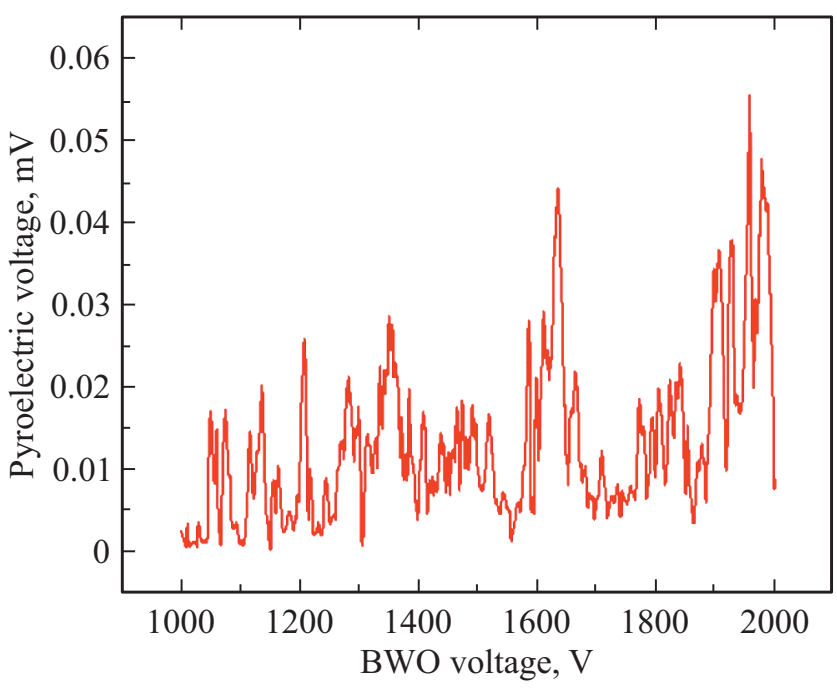

Рис. 9. Зависимость уровня выходного сигнала ЛОВ, снимаемого пироэлектрическим детектором, от ускоряющего напряжения.

В качестве примера, на рис. 9 представлена зависимость сигнала ЛОВ, регистрируемого пироэлектрическим детектором, от ускоряющего напряжения. К коммерчески произведенным ЛОВ часто прилагаются „калиброванные“ таблицы, где значениям ускоряющего напряжения соответствует мощность выходного сигнала. Но на практике дважды попасть в одну и ту же точку - невозможно, поэтому, прежде всего, необходимо одновременно с измерением отклика контролировать уровень сигнала источника (например, пироэлектриком). Далее, частично учесть переотражения на окнах криостата и внутри него позволяет рутениевый резистор, установленный на холодной плите криостата в непосредственной близости от исследуемого образца (рис. 7, $b$ ).
Другим источником погрешностей в таких измерениях является тепловой отклик болометрической структуры на нагрев подложки. В этом случае цепочка туннельных СИН-переходов, расположенная на краю чипа, может быть использована в качестве термометра. Регистрируя сигнал с такого термометра (рис. 7,c) и нормируя на него отклик болометрической матрицы, получаем более точную оценку спектрального отклика матрицы.

Перечисленные выше „источники искажения“ сигнала невозможно учесть при моделировании, т. к. необходима огромная вычислительная мощность, чтобы полностью воспроизвести весь приемо-передающий тракт.

Относительная неравномерность спектрального отклика (как в эксперименте, так и при моделировании) также связана с взаимным влиянием элементов матрицы друг на друга. На рис. 10 приведены параметры S11 элементов, расположенных в различных местах матрицы электрических малых антенн. Для сравнения на рис. 1

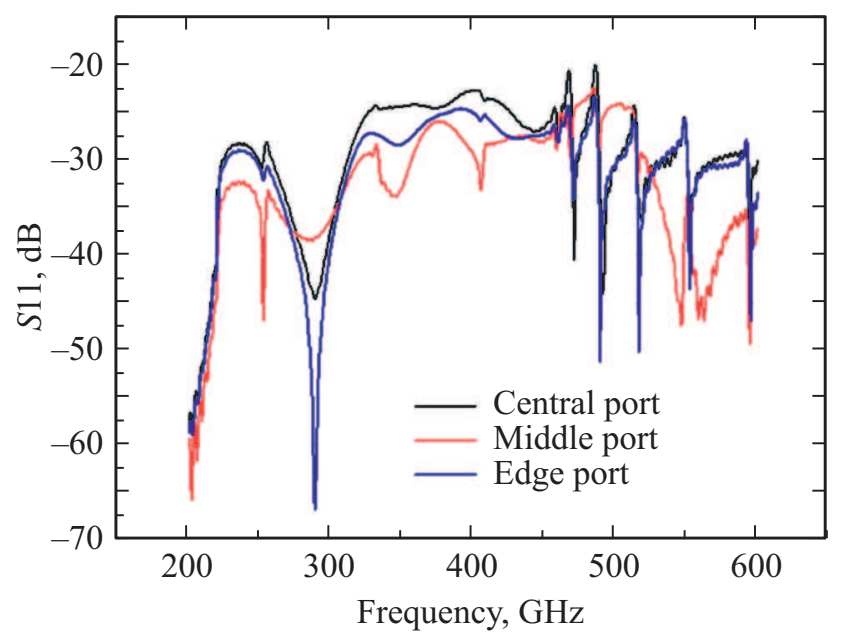

Рис. 10. Параметры S11 элементов матрицы электрически малых антенн. 


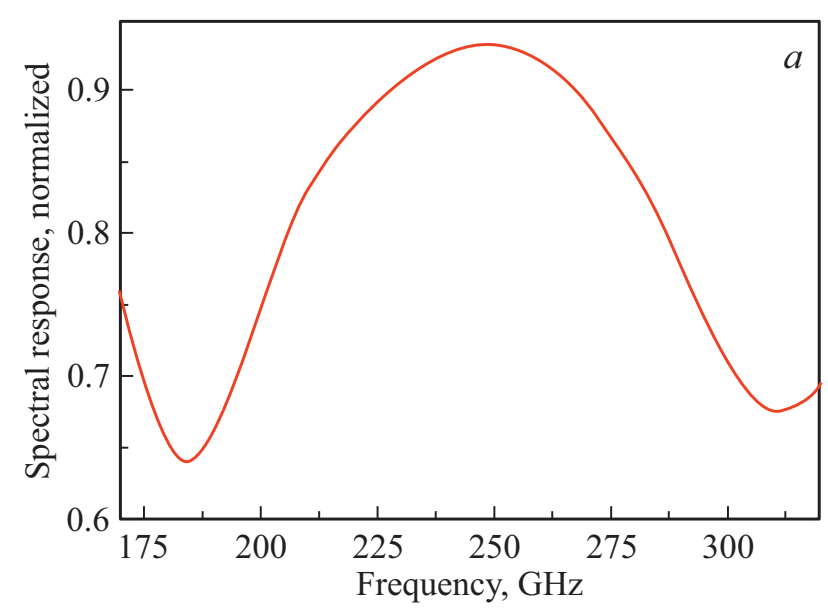

Рис. 11. Теоретический $(a)$ и экспериментально измеренный СИНИС-болометрами.

приведен смоделированный и экспериментально измеренный отклик одиночной кольцевой антенны [11].

\section{7. Заключение}

Были разработаны, изготовлены и экспериментально исследованы приемные матрицы субтерагерцевого диапазона с интегрированными СИНИС-болометрами. Для повышения точности оценки спектрального отклика приемной матрицы были смоделированы целые матрицы со всеми элементами, а в эксперименте использованы дополнительные опорные каналы для учета множественных переотражений. Такая тройная калибровка спектрального отклика позволила подтвердить необходимость моделирования всей матрицы целиком. Моделирование отдельного элемента в бесконечной матрице с периодическими граничными условиями соответствует фазированной антенной решетке и не описывает применяемую конструкцию, представляющую собой распределенный абсорбер.

\section{Финансирование работы}

Работа выполнена в рамках государственного задания ИРЭ РАН (№ 0030-2019-0003) и государственного задания ИПФ РАН (№ 0035-2019-0005). Изготовление и исследование образцов произведено с использованием уникальной научной установки (УНУ № 352529).

\section{Конфликт интересов}

Авторы заявляют, что у них нет конфликта интересов.

\section{Список литературы}

[1] И.И. Зинченко. Радиофизика 46, 641 (2003).

[2] P. Day, H.G. Leduc, C.D. Dowell, R.A. Lee, A. Turner, J. Zmuidzinas. J. Low Temp. Phys. 151, 477 (2008).

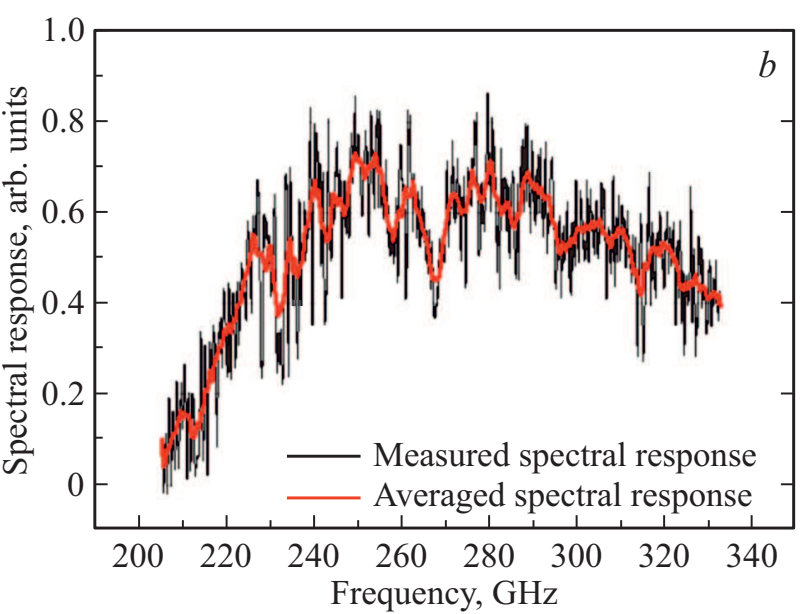

(b) [11] спектральный отклик одиночной кольцевой антенны с

[3] B. Westbrook, A. Cukierman, A. Lee, A. Suzuki, C. Raum, W. Holzapfel. J. Low Temp. Phys. 184, 74 (2016).

[4] P.K. Day, H.G. LeDuc, B.A. Mazin, A. Vayonakis, J. Zmuidzinas. Nature 425, 817 (2003).

[5] S. Masi, P. de Bernardis, A. Paiella, F. Piacentini, L. Lamagna, A. Coppolecchia, P.A.R. Ade, E.S. Battistelli, M.G. Castellano, I. Colantoni, F. Columbro, G. D’Alessandro, M. De Petris, S. Gordon, C. Magneville, P. Mauskopf, G. Pettinari, G. Pisano, G. Polenta, G. Presta, E. Tommasi, C. Tucker, V. Vdovin, A. Volpe, D. Yvon. J. Cosmol. Astropart. Phys. 2019, 7, 003 (2019).

[6] A. Tarasov, A.A. Gunbina, S. Mahashabde, R.A. Yusupov, A.M. Chekushkin, D.V. Nagirnaya, V.S. Edelman, G.V. Yakopov, V.F. Vdovin. IEEE Trans. Appl. Supercond. 30, 1 (2020).

[7] M. Tarasov, A. Sobolev, A. Gunbina, G. Yakopov, A. Chekushkin, R. Yusupov, S. Lemzyakov, V. Vdovin, V. Edelman. J. Appl. Phys. 125, 1 (2019).

[8] И.А. Девятов, П.А. Крутицкий, М.Ю. Куприянов. Письма в ЖЭТФ 84, 61 (2006).

[9] M. Tarasov, V. Edelman, S. Mahashabde, M. Fominsky, S. Lemzyakov, A. Chekushkin, R. Yusupov, D. Winkler, A. Yurgens. J. Phys.: Conf. Ser. 969, 012088 (2018).

[10] S. Mahashabde, A. Sobolev, A. Bengtsson, D. Andrén, M.A. Tarasov, M. Salatino, P. de Bernardis, S. Masi, L.S. Kuzmin. IEEE Trans. Terahertz Sci. Technol. 5, 145, (2015); S. Mahashabde, A.S. Sobolev, M.A. Tarasov, G.E. Tsydynzhapov, L.S. Kuzmin. IEEE Trans. Terahertz Sci. Technol. 5, 37 (2015).

[11] М.А. Тарасов, А.М. Чекушкин, Р.А. Юсупов, А.А. Гунбина, В.С. Эдельман. Радиотехника и электроника 65, 65 (2020).

[12] G. Yakopov, M. Tarasov, A. Gunbina, M. Mansfeld, R. Yusupov, V. Edelman, V. Vdovin. EPJ Web Conf. 195, 05014 (2018).

[13] R. Marques. IEEE Trans. Antennas Propag. 51,10 (2003)

[14] A.S. Sobolev, B. Beiranvand, A.M. Chekushkin, A.V. Kudryashov, M.A. Tarasov, R.A. Yusupov, A. Gunbina, V.F. Vdovin, V. Edelman. EPJ Web Conf. 195, (2018).

[15] С.А. Лемзяков, В.С. Эдельман. ПТЭ 59, 4, 146 (2016).

Редактор Е.Ю. Флегонтова 